Review

\title{
GroEL-Assisted Protein Folding: Does It Occur Within the Chaperonin Inner Cavity?
}

\author{
Victor V. Marchenkov and Gennady V. Semisotnov * \\ Institute of Protein Research, Russian Academy of Sciences, 142290, Russian Federation, Pushchino, \\ Moscow Region, Institutskaya street, 4, Russia; E-Mail: march@phys.protres.ru \\ * Author to whom correspondence should be addressed; E-Mail: nina@vega.protres.ru; \\ Tel. +7-495-632-7871; Fax: +7-495-632-7871
}

Received: 27 April 2009; in revised form: 8 May 2009 / Accepted: 11 May 2009 /

Published: 12 May 2009

\begin{abstract}
The folding of protein molecules in the GroEL inner cavity under the cochaperonin GroES lid is widely accepted as a crucial event of GroEL-assisted protein folding. This review is focused on the data showing that GroEL-assisted protein folding may proceed out of the complex with the chaperonin. The models of GroEL-assisted protein folding assuming ligand-controlled dissociation of nonnative proteins from the GroEL surface and their folding in the bulk solution are also discussed.
\end{abstract}

Keywords: chaperones; GroEL/ES chaperonin system; protein folding; protein aggregation

\section{Introduction}

In vitro experimental studies of protein unfolding and refolding reactions have led to an important conclusion that necessary and sufficient information on the spatial structure of proteins is included in their amino acid sequences [1,2]. However, the study of protein creation in vivo has revealed a number of cellular protein factors that are vital for the formation of the protein native conformation $[3,4]$. These protein factors, known as molecular chaperones [3], provide optimal conditions for protein folding in the cell which mostly prevent aggregation of nonfolded protein molecules. Besides, some chaperones participate in trans-membrane transport and degradation of proteins [5-8]. Many chaperones are members of a large group of heat shock proteins (hsp), whose biosynthesis in the cell is 
in effect enhanced by various cell stresses [9]. A number of chaperones are homo- or heterooligomers, consisting of many subunits usually combined in ring-like (toroidal) quaternary structures. Such complex quaternary structures are characteristic, for example, of hsp60 chaperones (chaperonins) and some representatives of small heat shock proteins [10-13].

Many studies have concentrated on the structural and functional properties of chaperonins, which assist protein folding in an ATP-dependent manner [10-13]. The GroE chaperonin system from Escherichia coli, consisting of two homooligomeric proteins GroEL and GroES, has been the object of numerous experimental and theoretical studies (see e.g. [12,14-32]). This system assists folding of various proteins through multiple rounds of binding and release of protein targets both in vivo and in vitro. GroEL shows no pronounced specificity towards nonnative protein targets and binds $40 \%$ of proteins from denatured extract of E. coli cells [31] and 30\% of newly synthesized proteins [24,25]. The electron microscopy [29] and crystallographic [20,21] data show that GroEL consists of 14 identical subunits of $57 \mathrm{kDa}$ each. The subunits are arranged into two stacked heptameric toroids, constituting a cylinder of $145 \AA$ height and $135 \AA$ diameter, with a central inner channel of $45 \AA$ in diameter (Figure 1).

Figure 1. Crystal structures of (a) GroEL chaperonin, (b) co-chaperonin GroES and (c) their complex. To show the inner cavity of GroEL (a) and "Anfinsen's cage" formed by the GroEL - GroES complex (c) front subunits of each GroEL ring and GroES have been removed. GroEL subunit domains ( $a$-apical, $i$-intermediate, and e-equatorial) and GroES subunits are shown by different colors. Black color shows exposed hydrophobic residues on the top of apical domains. The figure was plotted using the SwissPdb Viewer [109] and POV-Ray (www.povray.org) freeware and files 1OEL [20,21] and 1AON [32] which are available in PDB.

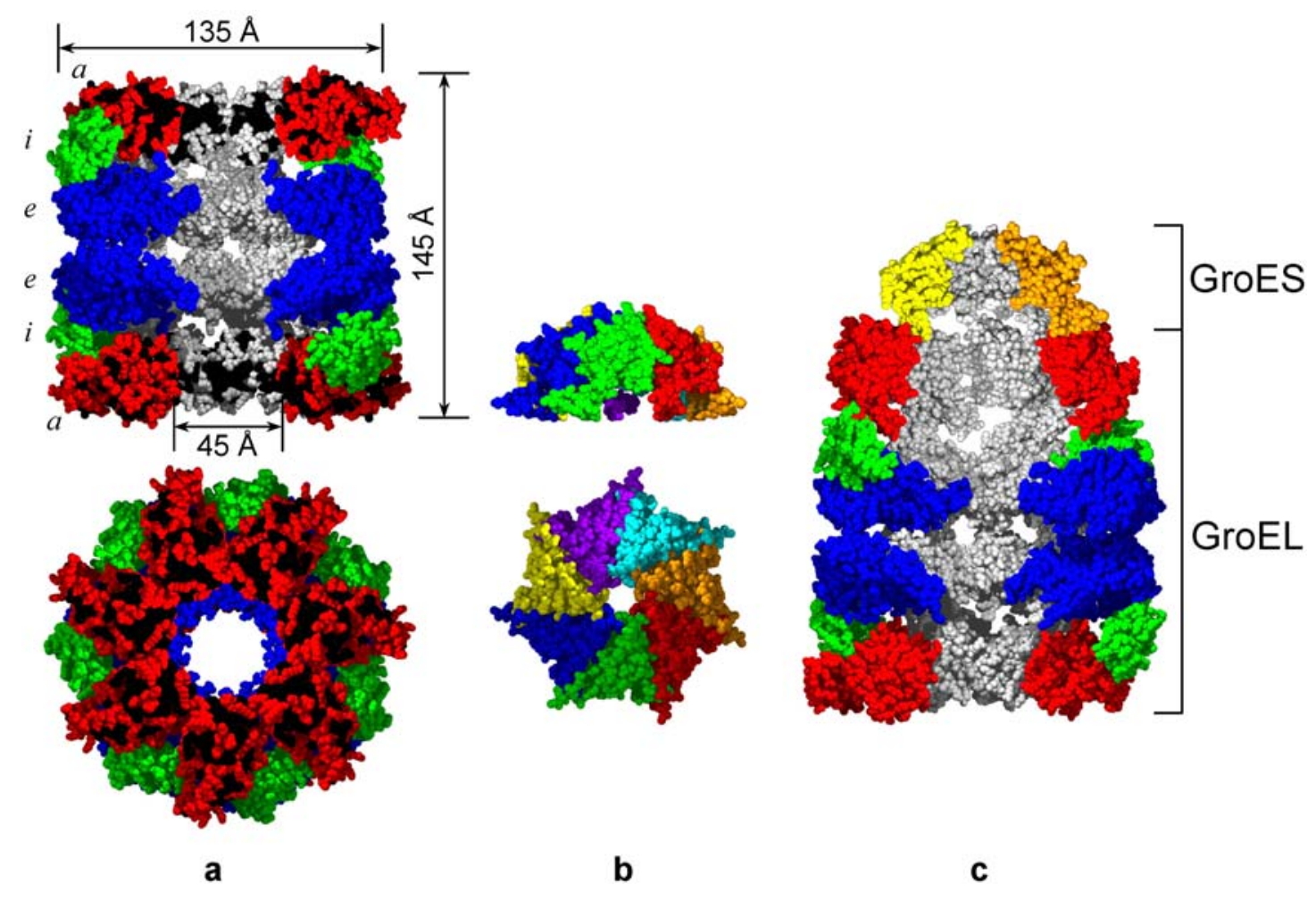


Each subunit of GroEL is composed of three clearly defined domains: apical (a), intermediate (i), and equatorial (e). The apical domains (191 - 376 amino acid residues) provide the binding sites for nonnative protein targets and co-chaperonin GroES [19,22,23,27,28]. The data from electron microscopy show that the nonnative protein target is bound at the edge of the inner channel $[19,22,27,28]$. At the same time the end-surfaces of the GroEL cylinder contain many exposed hydrophobic clusters (Figure 1) which may serve as additional sites for fixation of target proteins on the GroEL surface. The equatorial domain (6 - 133 and $409-523$ amino acid residues) is the largest domain of the GroEL subunit and provides the majority of intersubunit and interring contacts. The coordinates of $25 \mathrm{C}$ - and $5 \mathrm{~N}$-terminal amino acid residues of the GroEL subunit are not resolved by crystallography probably because of the high mobility of the residues [20]. However the data from electron microscopy and neutron diffuse scattering show that these terminal amino acid residues are located in the central channel of GroEL at the level of equatorial domains [22,27,28,30]. The ATP/ADP binding site is in the upper part of the equatorial domain on the inner surface of the central channel [18]. The intermediate domain of the GroEL subunit contains 89 amino acid residues (134 190 and 377 - 408) and connects the equatorial and apical domains providing large-scale conformational changes of the GroEL particle when interacting with ligands [18,22,32]. Cochaperonin GroES consists of 7 identical subunits arranged in a dome-like quaternary structure of $30 \AA$ height, $70-80 \AA$ in diameter and with a hole of $10 \AA$ in diameter (Figure 1) [26]. In the presence of Mg-ATP or Mg-ADP, GroES interacts with GroEL inducing large scale movements of the apical and intermediate domains due to which the extensive inner cavity chamber is formed under the GroES lid (Figure 1) [22,27,32-35]. The formation of this chamber (Anfinsen's cage) is the key structural argument for a suggestion that it is the place where the nonnative protein target bound with GroEL adopts the native structure in the absence of unfavorable contacts with other cellular components. This model of GroEL assisted protein folding has been worked out by several research groups during the recent 10 years and is widely accepted now [36-40]. Schematically the mechanism of protein folding in the GroEL inner cavity may be represented by the following main steps (Figure 2):

1. Nonnative protein binds with the preexisting asymmetrical complex GroEL:GroES in the inner cavity at the level of apical domains of the GroEL ring (trans ring) opposite to the ring bound to GroES.

2. Binding of the nonnative protein target induces an allosteric conformational change in the opposite ring leading to dissociation of GroES from GroEL.

3. GroES together with ATP binds once again with the GroEL ring containing nonnative protein (cis ring) and forms the so-called "Anfinsen's cage" preventing unfavorable contacts of the protein substrate.

4. Cooperative hydrolysis of 7 ATP molecules by the cis ring enhances its affinity to GroES and leads to some conformational changes in apical domains resulting in release of the nonnative protein target into the "Anfinsen's cage" for spontaneous folding in a state isolated from external medium.

5. Seven ATP molecules bind to the opposite “Anfinsen's cage" ring (trans ring). 
6. Cooperative hydrolysis of ATP by the trans ring leads to some allosteric conformational changes in the cis ring, which result in the dissociation of GroES and release of the native (folded) protein from the "Anfinsen's cage".

Figure 2. Scheme illustrating the mechanism of GroEL/ES assisted protein folding in the inner cavity ("Anfinsen's cage"). Non-native (unfolded) and native conformations of protein target are indicated as $\mathrm{U}$ and $\mathrm{N}$, respectively.

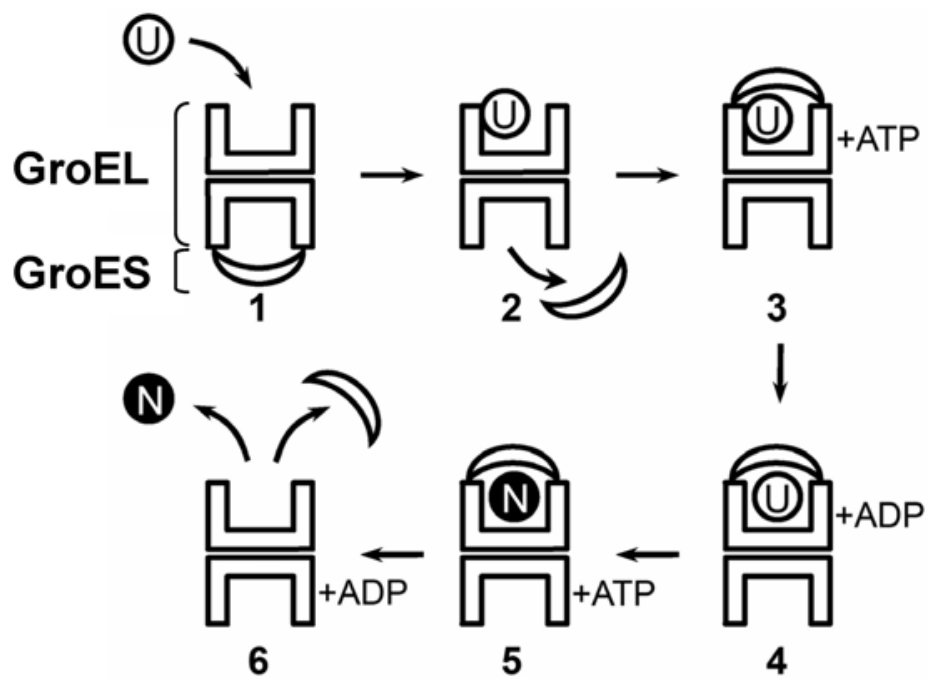

If one cycle is not enough for the final folding of the protein target, it reoccurs once again. Thus GroEL functions as a "molecular machine" controlled by its ligands, which induce allosteric conformational changes of the GroEL particle and define stages of the reaction cycle. This mechanism and its various modifications have so far undergone numerous experimental examinations and many experimental data have been satisfactorily explained within the framework of this model. At the same time, there are a number of experimental data showing that this mechanism of protein folding in the GroEL inner cavity is not universal yet. The present review is concentrated mainly on the data which are hardly intelligible supposing that GroEL-assisted protein folding occurs only by the above mechanism. An alternative mechanism of GroEL-assisted protein folding by the ligand-regulated binding-release of protein substrates and their folding in bulk solution is also discussed.

\section{GroEL Assists Folding of Large Proteins Whose Size Exceeds the Chaperonin Inner Cavity}

It is well established that GroEL is able to bind a wide range of nonnative or denatured proteins, as well as polypeptides of various sizes from $2 \mathrm{kDa}$ up to more than $100 \mathrm{kDa}$ both in vitro and in vivo [25,31,41-43]. The work of Houry et al. is an excellent example of identification of newly translated polypeptides tightly interacting with GroEL in vivo [25]. Using pulse radiolabelling, immunoprecipitation of GroEL with bound polypeptides, 2-D gel electrophoresis analysis and trypsinolysis combined with mass spectrometry, the authors demonstrated that $\sim 20 \%$ of polypeptides bound with GroEL have molecular mass exceeding $60 \mathrm{kDa}$. In particular the list of these high molecular mass polypeptides includes phosphate acetyltransferase $(77 \mathrm{kDa})$, tetrahydropteroyltriglutamate methyltransferase $(84 \mathrm{kDa})$, an RNA polymerase $\beta$-chain fragment $(150 \mathrm{kDa})$, and DNA 
gyrase subunit A $(97 \mathrm{kDa})$. The action of GroEL on the refolding of large proteins has not been thoroughly examined. The nonnative form of tailspike protein of phage P22 $(70 \mathrm{kDa})$ interacts with GroEL both in vitro [44] and in vivo [45]. At elevated temperature $\left(35^{\circ} \mathrm{C}\right)$ in the absence of ATP, GroEL effectively trapped protein refolding and reconstitution. However tailspike protein was released from GroEL by addition of ATP or without added ATP upon cooling to $25^{\circ} \mathrm{C}$, and native tailspike trimers were formed. The presence of GroES in addition to GroEL had no effect on reconstitution yield [44]. The $86 \mathrm{kDa} \alpha \beta$ heterodimer that is proposed to be an assembly intermediate of the $\alpha_{2} \beta_{2} \mathrm{E} 1$ enzyme of ketoacid dehydrogenase interacts with GroEL with 1:1 stoichiometry. The addition of Mg-ATP and GroES results in recovery of the active E1 tetramer [46]. The mechanism of GroELmediated folding of proteins, which are too large to be encapsulated under the GroES lid, is proposed for a large mitochondrial enzyme aconitase [47].The mitochondrial aconitase has molecular mass of 82 $\mathrm{kDa}$ that is too large to be encapsulated in the inner cavity of GroEL under the GroES lid, but the chaperonin assists the refolding of aconitase both in vivo and in vitro. The authors proposed that nonnative protein binds to the open (trans) ring of the GroEL-GroES complex and releases from the complex after binding and hydrolysis of ATP in the cis ring. The folding of the protein occurs in the free state (in bulk solution), and if the protein does not fold the cycle is repeated. The same mechanism was proposed for GroEL/GroES-assisted refolding of maltodextrin glucosidase (69 kDa monomeric Eshcherichia coli protein) [48]. To check the possibility for the protein to be bound to the trans ring of GroEL, the opposite ring was connected with GroES through a flexible polypeptide linker that was short enough to prevent the protein penetration into the "Anfinsen's cage" [49]. Nevertheless such an artificial GroEL-GroES system assists aconitase refolding with the same efficiency as the wild type system GroEL + GroES. Surprisingly this system revealed refolding assistance also for polypeptides of less molecular mass such as bacterial RuBisCo $(\sim 50 \mathrm{kDa})$ and mitochondrial malat dehydrogenase $(\sim 30 \mathrm{kDa})$. These proteins were proposed to be the so-called "stringent" substrates of GroEL, the productive folding of which requires encapsulation in the "Anfinsen's cage" [50,51]. From these experimental data it is possible to conclude that the GroEL ring opposite to the GroES bound one can assist as well the folding of not only large proteins but also proteins which can be encapsulated in the chaperonin cavity.

\section{GroEL Is Able to Assist Protein Folding in the Absence of GroES, ATP Hydrolysis and Double Ring Structure}

GroEL functioning as a molecular chaperone is provided by interaction with a number of ligands: $\mathrm{K}$ and $\mathrm{Mg}$ ions, adenine nucleotides (ADP and ATP) and co-chaperonin GroES [10,12,18,29,31,32, 50,52]. However, it is impossible to make a general conclusion on the ligand involvement in the functioning of GroEL. The presence of GroEL either alone or in addition to ADP or ATP can be enough for effective GroEL-assisted protein folding [13,53-56], while the interaction with GroES usually enhances this reaction [54,57,58]. It seems likely that the ligands decrease the affinity of GroEL to nonnative protein targets with the efficiency increasing pursuant to $\mathrm{Mg}-\mathrm{ADP}<\mathrm{Mg}-\mathrm{ATP}<$ Mg-ATP-GroES [58]. Studies of the protein refolding kinetics in the presence of GroEL and its ligands show that the chaperonin interacts with early kinetic intermediates having properties of the "molten globule" [19,59-61] and slows down their transition to the native state [58,62,63]. This interaction 
occurs very fast [62-64] and probably depends on the "hydrophobicity" and charge of the protein target [23,64-69] as well as on experimental conditions. If the lifetime of the GroEL complex with early protein refolding intermediates is comparably short (the so-called "transient interaction"), the refolding of the intermediates can be observed in the absence of ligands [62-64,70]. However, if the interaction of these intermediates with GroEL is strong (the lifetime of the complex is large), their refolding to the native state seems to be "arrested" during the observation time and appears only after an addition of GroEL ligands (see, for example, [50,71]), which likely decrease the GroEL affinity to protein refolding intermediates, and they can adopt native structure during the observation time. The double ring structure of GroEL is not required for assisting the protein folding as well. GroEL single ring conformation [72,73] and even isolated apical domains [74] are able to assist the protein folding. The mammalian mitochondrial chaperonin Hsp60 exists in a single ring conformation [75] but facilitates the refolding of ribulose-biphosphate carboxylase in an ATP-dependent manner [76,77]. The GroEL mutant with four amino acid substitutions in the equatorial interring surface also exists in a single ring conformation (SR1), but is functionally inactive probably due to its inability to release GroES [40,72]. However, the change of experimental conditions [57] or the change of mutations [78] lead to activation of the GroEL single ring conformation toward assisting the refolding of some proteins. Moreover, the $34 \mathrm{kDa}$ proteolytic fragment of GroEL (residues $150-456$ ) as well as the 50 $\mathrm{kDa}$ fragment of the Thermus thermophilus chaperonin are able to assist refolding of denatured rhodanese in the absence of GroES and ATP [79,80]. Fersht with co-workers constructed plasmids encoding the GroEL apical domain alone (residues 191 - 376) and some of its fragments and expressed them in E. coli cells [74]. They found that the GroEL apical domain alone and especially its C-terminal truncated fragments assist the refolding of rhodanese and cyclophilin, functioning as "minichaperones" in the absence of ATP. It may be suggested from the above experimental data that the inner cavity of GroEL is not directly related to its chaperoning activity. At the same time there are many evidences that the inner cavity participates in the GroEL-assisted protein folding (see reviews $[10,12,81,82])$. From recent publications it is possible to indicate the following:

Tang et al. [83] modulated the volume of the GroEL central cavity by a step-wise extension or reduction of the $\mathrm{C}$-terminal sequence protruding from the equatorial domains into the cavity. They found that a change of the cavity volume resulted in both a change in the capacity of protein "encapsulation" and a change of the target protein refolding rate. Moreover, in a later publication they showed in vivo that a change of the GroEL cavity volume or charge affects cell viability and intracellular protein folding [84]. On the other hand, Farr et al. [85] reported that the effect of the GroEL C-terminal sequence extension on the protein folding rate may be due to the change in the ATP-ase activity. In addition the influence of the GroEL C-terminal sequence length on the chaperonin structure and its affinity to the substrate proteins can not be excluded either (our comment). Another set of the evidences goes from the mass-spectrometry and electron microscopy data indicating the place of target protein within the GroEL particle. Ester van Duijn et al. performed a number of massspectrometry studies of macromolecular complexes involved in the chaperonin-assisted protein folding. They found that the complex GroEL/protein substrate/co-chaperonin contains one GroEL particle, one monomeric protein substrate molecule and one co-chaperonin molecule [34]. However, they were unable to distinguish where the substrate molecule was bound either in the cis or trans ring of the complex. In another work the immersion of some substrate proteins into the GroEL particle was 
studied using native mass spectrometry combined with ion mobility mass spectrometry (IM-MS) [35]. It was shown that the complexes of GroEL with a number of denatured protein substrates have dimensions ("collision cross sections") similar to those of free GroEL which shows that these genuine substrates are buried within the chaperonin cavity. In contrast, the binding of native BSA to GroEL resulted in a significantly larger dimension of the complex in comparison with free GroEL. The authors concluded that the binding of the native BSA ligand to GroEL is nonspecific and occurs on the top of the apical domains out of the cavity. Using the same approach the authors showed that the binding of GroES to the pre-existing GroEL/protein substrate complex increases the complex dimension ("collision cross section") only by the amount of GroES, confirming deep immersion of the protein substrate into the cavity [35].

A more obvious location of the substrate protein molecule within the GroEL/co-chaperonin complex was confirmed by cryo-electron microscopy. However, the data obtained for different chaperonins and protein substrates are somewhat conflicting. In early publications it was reported that the non-native protein substrate is placed in the trans ring of the GroEL-GroES complex for both Escherichia coli [22] and Thermus thermophilus [27] chaperonin systems. Recent publications show that the protein substrate molecule is placed in the cis ring of the GroEL/co-chaperonin complex for both Escherichia coli GroEL with the bacteriophage T4 GroES - analog (gp31) system [33] and Thermus thermophilus GroEL-GroES system [86]. This contradiction may be a result of different techniques used for preparation of the samples, or the co-chaperonin can distinguish what GroEL ring must be the cis one in dependence on the substrate protein. In any case, the electron microscopy data give us visual information that the protein substrate can occupy both GroEL rings simultaneously and can be encapsulated in the cavity $[33,86]$.

The importance of the ring-like organization of the GroEL particle and hence its inner cavity is somewhat supported by the data that the protein target can undergo multiple partial unfolding to overcome misfolded conformations and enhance the yield of a correct folded conformation in accordance with "the iterative annealing mechanism" [87]. However this mechanism is still argued due to experimental difficulties to distinguish between protein substrate unfolding as a result of its binding to GroEL and as a result of the shift of the equilibrium toward a more-unfolded protein state that may preferentially bind to GroEL (see the discussion in the review by Horwich et al. [12]).

\section{GroEL Is Able to Bind Simultaneously More Than One Polypeptide Substrate or Two GroES Molecules}

The stoichiometry of the GroEL-polypeptide complex is poorly studied and the existing data are again conflicting. The crystal structure of GroEL with a bound small polypeptide $(\sim 2 \mathrm{kDa})$ in the absence of nucleotides shows that small polypeptides bind to each of 14 subunits in the region of a pair of parallel helices at the top of the apical domain [41]. The same polypeptide binding sites have been found in the isolated apical domain of GroEL [74,78]. Interestingly, the polypeptides are bound to GroEL at the same places as the GroES molecule [32,41]. As for large polypeptides such as proteins, the data are contradictory. Titration calorimetry experiments on the GroEL binding with two denatured proteins (pepsin at $\mathrm{pH} 7$ and reduced $\alpha$-lactalbumin) show 1:1 stoichiometry for the both proteins [88]. The equimolar stoichiometry of the nonnative protein target bound to the GroEL particle was reported 
also for a mutant form of subtilisin [89]. However, for maltose-binding protein [55] and for mutants of staphylococcal nuclease [90] the stoichiometry of the GroEL/substrate complex was demonstrated to be 1:2. GroEL was reported to bind up to 4-5 molecules of nonnative barnase [91] or mutant forms of dihydrofolate reductase [92]. Chuang and co-workers reported that GroEL saturated with a nonnative $\alpha \beta$ heterodimer of $\alpha$-ketoacid dehydrogenase up to $1: 1$ stoichiometry is able to bind additionally a stoichiometric amount of denatured lysozyme [46]. Young and co-workers have shown that integral membrane proteins can be soluble without any detergent by binding to the GroEL tetradecameric particle. In particular, after dialysis of detergent-solubilized bacteriorhodopsin in the presence of GroEL, it was found that GroEL binds two molecules of the protein at saturation [93]. In the other study they analyzed solubilization of a membrane protein $\lambda$-holin by GroEL and found that the protein saturates the chaperonin at six molecules per tetradecameric GroEL particle, whereas the protein variant missing two N-terminal residues forms a hypersolubilization complex with up to 350 holin molecules per GroEL particle. In the presence of ATP or its nonhydrolyzed analog AMP-PNP no solubilization was observed, while in the presence of ADP slow precipitation of holin molecules was detected [94]. Stoichiometry measurements of GroEL-protein target complexes may be complicated by polypeptide impurities tightly bound with GroEL [95]. We purified GroEL from such impurities according to the published protocol [96] and measured the stoichiometry of GroEL complexes with denatured pepsin, reduced $\alpha$-lactalbumin, lysozyme and bovine serum albumin using fluorescence anisotropy titration and size-exclusion chromatography. Two protein molecules per GroEL tetradecamer were found to be bound for all of these proteins (our unpublished data). The data from mass spectrometry [34] and cryo-electron microscopy [33] also show 1:2 stoichiometry of the GroEL/protein substrate binary complex. The symmetrical GroEL double ring structure is able to bind not only more than one polypeptide target but under certain conditions two molecules of its cochaperonin GroES [97-102]. Thus, the GroEL complex with GroES can exist in two forms: asymmetrical and symmetrical $[98,99]$.

\section{GroEL-GroES Complex Does Not Maintain Interactions with Denatured Proteins}

Most experiments on the release of target proteins from the GroEL-GroES complex are performed using protein refolding systems. However, in such cases it is difficult to distinguish the events of formation of protein native structure and protein dissociation from the complex because native protein usually does not interact with GroEL. However, if the formation of protein native structure is inhibited by experimental conditions (the presence of disulfide bond reducing agents, nonnative $\mathrm{pH}$ or temperature) or by mutations or truncation of the protein chains it is possible to study more easily the binding and release of protein targets. Using size-exclusion chromatography Yoshida and co-workers showed that GroEL in the absence of its ligands tightly binds pepsin denatured at neutral $\mathrm{pH}$. Mg-ATP decreases GroEL affinity to the protein target while the binding of GroES in the presence of Mg-ATP leads to practically full dissociation of denatured pepsin from the GroEL-GroES complex [88]. A similar result was obtained by us later [67]. Moreover, in some cases when denatured proteins are strongly aggregated, the GroEL-GroES complex is not able to prevent their aggregation as it was shown by us for reduced lysozyme [103] and for truncated variants of elongation factor EF-2 [104]. These data challenge the proposition that nonnative proteins of appropriate molecular mass must be 
enclosed in the cis chamber of the GroEL-GroES complex. Indeed, the timing of a GroEL/GroES assisted protein folding reaction shows that at $23{ }^{\circ} \mathrm{C}$ the polypeptide bound with GroEL is released into the closed chamber within a second after a cis complex formation [105]. The substrate protein then lives about $10 \mathrm{~s}$ in the cavity under GroES lid till GroES can be discharged by the binding of ATP in the trans ring (Figure 2) [101,106,107]. Thus, the equilibrium must be strongly shifted towards the GroEL/substrate protein/GroES ternary complex. In accord with this, if protein target cannot adopt the rigid (native) structure it must be mainly involved in the ternary complex and cannot be separated from the complex by size-exclusion chromatography [67,88] or aggregated $[103,104]$. On the contrary, these data support the proposal that interaction of GroES with the binary GroEL-nonnative protein complex decreases the affinity of the chaperonin to the protein target may be due to the competition between co-chaperonin and nonnative proteins for the same binding sites.

\section{The Models of GroEL-Assisted Protein Folding out of the Chaperonin Inner Cavity}

The experimental data reviewed herein are hardly understandable assuming that the protein target can be folded only in the inner cavity of GroEL under the GroES lid. Therefore some investigators suggest that GroEL assists large proteins folding by a "trans" mechanism [47-49]. This mechanism proposes that protein folding occurs in bulk solution while the release of the protein target from GroEL is due to allosteric conformational changes in the "trans" ring bound with protein substrate which are induced by GroES binding in the "cis" ring. Recently we proposed some model by which GroEL may assist the folding of various proteins by time- or ligand-controlled multiple rounds of binding-release of the protein targets and their final folding in bulk solution [108] (Figure 3).

Figure 3. Scheme illustrating the mechanism of GroEL functioning as a molecular chaperone under the assumption of the target protein folding in the free state (in bulk solution) [108]. Different symbols indicate different polypeptides which are bound by GroEL with different association constants. Nonnative (unfolded) and native conformations of polypeptides are indicated as $\mathrm{U}$ and $\mathrm{N}$, respectively.

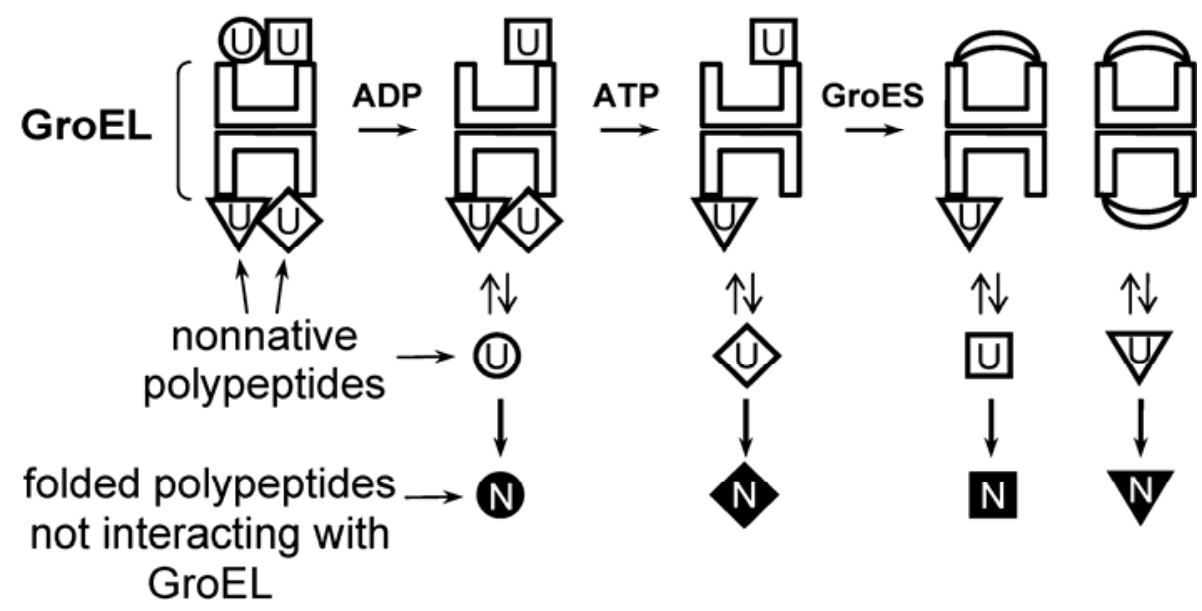

The model is based on the following principles: The GroEL particle is able to bind various polypeptides lacking rigid tertiary structure on both of its barrel ends including the tops of the cavities. 
The number of bound polypeptides depends on their size while the affinity to GroEL is determined by their hydrophobicity and charge. Thus, the concentration of nonnative polypeptides in bulk solution (in the free state) drops abruptly which reduces the probability of their nonspecific interactions. Weakly bound polypeptides can sometimes dissociate from the GroEL surface and adopt native (rigid) conformation in bulk solution or interact with other cellular factors even in the absence of GroEL ligands.

The ligands (Mg-ADP, Mg-ATP and co-chaperonin GroES) weaken to a different extent the interaction of nonnative polypeptides with GroEL. As a result the lifetime of polypeptides in the free state and hence the probability for them to adopt native structure or to be bound to other cellular factors preventing their nonspecific association (e.g., to other chaperones) is enhanced.

The model does not require the protein target encapsulation in the "Anfinsen's cage" where its folding may be problematic and does not impose strict limitations on the target size and stoichiometry of the complexes with GroEL. At the same time such a mechanism provides the GroEL function to prevent nonspecific intermolecular association of polypeptides lacking a rigid tertiary structure. Thus, GroEL functioning as a molecular chaperone is restricted by binding strongly "hydrophobic" states of polypeptides resulting in lowering their concentration in bulk solution and assisting their folding through ligand- or time-dependent multiple binding-release rounds. GroEL ligands prevent formation of long-living chaperonin-substrate complexes which are not advantageous for fast recovery of cells after the stress.

\section{Conclusions}

It is now well known that protein (re)folding is often complicated by nonspecific intermolecular interactions of protein molecules in intermediate states with high exposure of hydrophobic groups. These interactions may lead to a slowing down the protein native structure formation or, under strongly nonpermissive conditions, to the formation of large aggregates which practically inhibit protein folding. Nonspecific intermolecular interactions upon protein folding in vitro can be essentially reduced by choosing appropriate refolding conditions. Among them the following seems to be most effective. The first is temperature drop leading to weakening of hydrophobic attraction. The second is a decrease of the protein concentration that reduces the probability of intermolecular association of protein molecules. The third is enhancement of the electrostatic repulsion of aggregation-prone intermediates that can be achieved either by decreasing ionic strength of the renaturing mixture or by changing $\mathrm{pH}$. In some cases the addition of denaturants (urea or $\mathrm{GuHCl}$ ) or low molecular weight compounds (such as arginine, glycerol, sucrose) to the renaturing mixture is also effective. Very often these simple operations allow overcoming the problems accompanying protein folding in vitro. However, in the cell the protein aggregation is controlled by chaperones. The majority of chaperones is able to recognize polypeptides lacking rigid tertiary structure and to prevent their nonspecific association (see, for example, $[10,11,13]$ ) . Many chaperones work using the ligands which provide regulation of binding/release rounds of the substrate polypeptides. Among them there are monomeric or dimeric chaperones with hydrophobic pockets for substrate polypeptides binding as well as more complex oligomeric chaperones which bind polypeptide substrates by multivalent interactions with exposed hydrophobic clusters of their subunits [11]. The quaternary structure of the latter is usually 
formed as one or two ring-shaped oligomers consisting of several homo- or hetero-subunits forming a distinct inner cavity. Despite of similar organization of the quaternary structure of these chaperones and the presence of inner cavity, the interaction with GroES-like co-chaperones has been found only for GroEL-like representatives of this group of chaperones. The inner cavity of chaperones lacking cochaperones seems to be less essential for protein folding than in the case of GroEL. Indeed a partial occlusion of both cavities in the eukaryotic chaperonin CCT with antibody has no effect on the rate and yield of substrate protein folding [110]. A variety of chaperones may be divided according to the mechanisms of their functioning in the following groups. The first group includes small heat shock proteins and $\alpha$-crystalline which prevent protein aggregation in an ATP-independent manner [111,112]. These oligomeric proteins, consisting of $12-43 \mathrm{kDa}$ subunits with different multimeric organization, bind denatured proteins but their release from small heat shock proteins is very slow. It has been proposed that their main function is to prevent aggregation of denatured proteins and to form some reservoir for other chaperone systems to renature the bound proteins [111]. Small heat shock proteins do not show substrate specificity and possibly bind polypeptide substrates on the external surface of the multimer [11]. The second group represents monomeric and dimeric chaperones whose functioning is dependent on the ADP/ATP ligands and protein cofactors. Molecular chaperones of the Hsp70 family not only bind substrate proteins and prevent their aggregation but provide their subsequent folding (see reviews [10,11]). They are composed of two functional parts one of which binds ADP or ATP and possesses ATP-ase activity, whereas the other one binds substrate polypeptides. Chaperones of this family are usually functioning in cooperation with two chaperones of the Hsp40 family [10]. ATP binding induces some conformational changes in Hsp70 resulting in decreasing the chaperone affinity for nonnative substrate proteins [113]. Polypeptide substrates released from the Hsp70 chaperones undergo kinetic partitioning between folding to native conformation, aggregation, rebinding to the chaperone and binding to other components of a multidirectional protein folding network. Similar activities have been shown for dimeric chaperones of the Hsp90 family [11]. They also use adenine nucleotides and interaction with other protein cofactors and chaperones to provide effective protein folding. The third group of chaperones represents oligomeric ring-like organized complexes with distinct inner cavities. To execute their functions, these chaperones require adenine nucleotides and some of them also GroES-like co-chaperonins. Members of the Hsp100 family are six-subunit ring-like complexes which contain ATP and substrate polypeptide binding sites and are able to substitute the Hsp70 chaperone systems in effective protein refolding [11,114]. The other members of this group are the so-called chaperonins which are organized as multimeric double ring complexes with two extensive inner cavities. Among them it is possible to discriminate GroEL-like chaperonins having GroES-like co-chaperonins and those which have no cochaperonins [11-13,15]. Nevertheless both types of chaperonins assist protein folding both in vivo and in vitro by multiple ATP-dependent repeated binding-release of substrate polypeptides [12]. Up to date only for the GroEL/co-chaperonin complex the substrate protein was found inside the cavity under the GroES lid. The above analysis of published data raises the question about the importance of inner cavities of oligomeric chaperones as the universal place where protein substrates are prevented from aggregation and can adopt native structure. Further studies of the properties of monomeric and oligomeric chaperones from different organisms and cellular compartments will allow us to clarify general principles of their participation in the processes of protein creation and transport in the cell. 


\section{Acknowledgements}

We thank members of our group Svetlana Yu. Marchenkova, Nataliya Yu. Marchenko and Bogdan S. Melnik for their help in preparing the text and reference list. This work was supported by grants from HHMI (55,005,607), Russian Program for Scientific Schools (2,791. 2008. 4) and Russian Foundation for Basic Research (grant № 09-04-00768-a).

\section{References and Notes}

1. Anfinsen, C.B. Principles that govern the folding of protein chains. Science 1973, 181, 223-230.

2. Seckler, R.; Jaenicke, R. Protein folding and protein refolding. FASEB J. 1992, 6, 2545-2552.

3. Ellis, J. Proteins as molecular chaperones. Nature 1987, 328, 378-379.

4. Gething, M.J.; Sambrook, J. Protein folding in the cell. Nature 1992, 355, 33-45.

5. Bochkareva, E.S.; Lissin, N.M.; Girshovich, A.S. Transient association of newly synthesized unfolded proteins with the heat-shock GroEL protein. Nature 1988, 336, 254-257.

6. Hayes, S.A.; Dice, J.F. Roles of molecular chaperones in protein degradation. J. Cell Biol. 1996, 132, 255-258.

7. Kandror, O.; Sherman, M.; Goldberg, A. Rapid degradation of an abnormal protein in Escherichia coli proceeds through repeated cycles of association with GroEL. J.Biol.Chem. 1999, 274, 37743-37749.

8. Gething, M.J. Protein folding. The difference with prokaryotes. Nature 1997, 388, 329-331.

9. Lindquist, S.; Craig, E.A. The heat-shock proteins. Annu. Rev. Genet. 1988, 22, 631-677.

10. Bukau, B.; Horwich, A.L. The Hsp70 and Hsp60 chaperone machines. Cell 1998, 92, 351-366.

11. Fink, A.L. Chaperone-mediated protein folding. Physiol. Rev. 1999, 79, 425-449.

12. Horwich, A.L.; Fenton, W.A.; Chapman, E.; Farr, G.W. Two families of chaperonin: physiology and mechanism. Annu. Rev. Cell Dev. Biol. 2007, 23, 115-145.

13. Jaenicke, R. What does protein refolding in vitro tell us about protein folding in the cell? Philos. Trans. R. Soc. Lond B Biol. Sci. 1993, 339, 287-294.

14. Bhutani, N.; Udgaonkar, J.B. A thermodynamic coupling mechanism can explain the GroELmediated acceleration of the folding of barstar. J. Mol. Biol. 2000, 297, 1037-1044.

15. Braig, K. Chaperonins. Curr. Opin. Struct. Biol. 1998, 8, 159-165.

16. Feltham, J.L.; Gierasch, L.M. GroEL-substrate interactions: molding the fold, or folding the mold? Cell 2000, 100, 193-196.

17. Hartl, F.U. Protein folding. Secrets of a double-doughnut. Nature 1994, 371, 557-559.

18. Boisvert, D.C.; Wang, J.; Otwinowski, Z.; Horwich, A.L.; Sigler, P.B. The 2.4 A crystal structure of the bacterial chaperonin GroEL complexed with ATP gamma S. Nat. Struct. Biol. 1996, 3, 170-177.

19. Braig, K.; Simon, M.; Furuya, F.; Hainfeld, J.F.; Horwich, A.L. A polypeptide bound by the chaperonin groEL is localized within a central cavity. Proc. Natl. Acad. Sci. USA 1993, 90, 3978-3982. 
20. Braig, K.; Otwinowski, Z.; Hegde, R.; Boisvert, D.C.; Joachimiak, A.; Horwich, A.L.; Sigler, P.B. The crystal structure of the bacterial chaperonin GroEL at $2.8 \AA$. Nature 1994, 371, 578-586.

21. Braig, K.; Adams, P.D.; Brunger, A.T. Conformational variability in the refined structure of the chaperonin GroEL at 2.8 Å resolution. Nat. Struct. Biol. 1995, 2, 1083-1094.

22. Chen, S.; Roseman, A.M.; Hunter, A.S.; Wood, S.P.; Burston, S.G.; Ranson, N.A.; Clarke, A.R.; Saibil, H.R. Location of a folding protein and shape changes in GroEL-GroES complexes imaged by cryo-electron microscopy. Nature 1994, 371, 261-264.

23. Fenton, W.A.; Kashi, Y.; Furtak, K.; Horwich, A.L. Residues in chaperonin GroEL required for polypeptide binding and release. Nature 1994, 371, 614-619.

24. Horwich, A.L.; Low, K.B.; Fenton, W.A.; Hirshfield, I.N.; Furtak, K. Folding in vivo of bacterial cytoplasmic proteins: role of GroEL. Cell 1993, 74, 909-917.

25. Houry, W.A.; Frishman, D.; Eckerskorn, C.; Lottspeich, F.; Hartl, F.U. Identification of in vivo substrates of the chaperonin GroEL. Nature 1999, 402, 147-154.

26. Hunt, J.F.; Weaver, A.J.; Landry, S.J.; Gierasch, L.; Deisenhofer, J. The crystal structure of the GroES co-chaperonin at $2.8 \AA$ resolution. Nature 1996, 379, 37-45.

27. Ishii, N.; Taguchi, H.; Sasabe, H.; Yoshida, M. Folding intermediate binds to the bottom of bullet-shaped holo-chaperonin and is readily accessible to antibody. J. Mol. Biol. 1994, 236, 691-696.

28. Langer, T.; Pfeifer, G.; Martin, J.; Baumeister, W.; Hartl, F.U. Chaperonin-mediated protein folding: GroES binds to one end of the GroEL cylinder, which accommodates the protein substrate within its central cavity. EMBO J. 1992, 11, 4757-4765.

29. Roseman, A.M.; Chen, S.; White, H.; Braig, K.; Saibil, H.R. The chaperonin ATPase cycle: mechanism of allosteric switching and movements of substrate-binding domains in GroEL. Cell 1996, 87, 241-251.

30. Thiyagarajan, P.; Henderson, S.J.; Joachimiak, A. Solution structures of GroEL and its complex with rhodanese from small-angle neutron scattering. Structure. 1996, 4, 79-88.

31. Viitanen, P.V.; Gatenby, A.A.; Lorimer, G.H. Purified chaperonin 60 (groEL) interacts with the nonnative states of a multitude of Escherichia coli proteins. Protein Sci. 1992, 1, 363-369.

32. $\mathrm{Xu}, \mathrm{Z}$;; Horwich, A.L.; Sigler, P.B. The crystal structure of the asymmetric GroEL-GroES$(\mathrm{ADP})_{7}$ chaperonin complex. Nature 1997, 388, 741-750.

33. Clare, D.K.; Bakkes, P.J.; van Heerikhuizen H.; van der Vies, S.M.; Saibil, H.R. Chaperonin complex with a newly folded protein encapsulated in the folding chamber. Nature 2009, 457, 107-110.

34. van Duijn, E.; Bakkes, P.J.; Heeren, R.M.; van den Heuvel, R.H.; van Heerikhuizen, H.; van der Vies, S.M.; Heck, A.J. Monitoring macromolecular complexes involved in the chaperoninassisted protein folding cycle by mass spectrometry. Nat. Methods 2005, 2, 371-376.

35. van Duijn, E.; Barendregt, A.; Synowsky, S.; Versluis, C.; Heck, A.J. Chaperonin complexes monitored by ion mobility mass spectrometry. J. Am. Chem. Soc. 2009, 131, 1452-1459.

36. Ellis, R.J.; Hartl, F.U. Principles of protein folding in the cellular environment. Curr. Opin. Struct. Biol. 1999, 9, 102-110. 
37. Martin, J.; Mayhew, M.; Langer, T.; Hartl, F.U. The reaction cycle of GroEL and GroES in chaperonin-assisted protein folding. Nature 1993, 366, 228-233.

38. Mayhew, M.; da Silva, A.C.; Martin, J.; Erdjument-Bromage, H.; Tempst, P.; Hartl, F.U. Protein folding in the central cavity of the GroEL-GroES chaperonin complex. Nature 1996, 379, 420-426.

39. Radford, S.E. GroEL: More than Just a folding cage. Cell 2006, 125, 831-833.

40. Weissman, J.S.; Rye, H.S.; Fenton, W.A.; Beechem, J.M.; Horwich, A.L. Characterization of the active intermediate of a GroEL-GroES-mediated protein folding reaction. Cell 1996, 84, 481-490.

41. Chen, L.; Sigler, P.B. The crystal structure of a GroEL/peptide complex: plasticity as a basis for substrate diversity. Cell 1999, 99, 757-768.

42. Ewalt, K.L.; Hendrick, J.P.; Houry, W.A.; Hartl, F.U. In vivo observation of polypeptide flux through the bacterial chaperonin system. Cell 1997, 90, 491-500.

43. Lau, C.K.; Churchich, J.E. Binding of polylysine to GroEL. Inhibition of the refolding of mMDH. Biochim. Biophys. Acta 1999, 1431, 282-289.

44. Brunschier, R.; Danner, M.; Seckler, R. Interactions of phage P22 tailspike protein with GroE molecular chaperones during refolding in vitro. J. Biol. Chem. 1993, 268, 2767-2772.

45. Gordon, C.L.; Sather, S.K.; Casjens, S.; King, J. Selective in vivo rescue by GroEL/ES of thermolabile folding intermediates to phage P22 structural proteins. J. Biol. Chem. 1994, 269, 27941-27951.

46. Song, J.L.; Wynn, R.M.; Chuang, D.T. Interactions of GroEL/GroES with a heterodimeric intermediate during $\alpha_{2} \beta_{2}$ assembly of mitochondrial branched-chain $\alpha$-ketoacid dehydrogenase. cis Capping of the native-like $86-\mathrm{kDa}$ intermediate by GroES. J. Biol. Chem. 2000, 275, 22305-22312.

47. Chaudhuri, T.K.; Farr, G.W.; Fenton, W.A.; Rospert, S.; Horwich, A.L. GroEL/GroES-mediated folding of a protein too large to be encapsulated. Cell 2001, 107, 235-246.

48. Paul, S.; Punam, S.; Chaudhuri, T.K. Chaperone-assisted refolding of Escherichia coli maltodextrin glucosidase. FEBS J. 2007, 274, 6000-6010.

49. Farr, G.W.; Fenton, W.A.; Chaudhuri, T.K.; Clare, D.K.; Saibil, H.R.; Horwich, A.L. Folding with and without encapsulation by cis- and trans-only GroEL-GroES complexes. EMBO J. 2003, 22, 3220-3230.

50. Goloubinoff, P.; Christeller, J.T.; Gatenby, A.A.; Lorimer, G.H. Reconstitution of active dimeric ribulose bisphosphate carboxylase from an unfolded state depends on two chaperonin proteins and Mg-ATP. Nature 1989, 342, 884-889.

51. Miller, A.D.; Maghlaoui, K.; Albanese, G.; Kleinjan, D.A.; Smith, C. Escherichia coli chaperonins cpn60 (groEL) and cpn10 (groES) do not catalyse the refolding of mitochondrial malate dehydrogenase. Biochem. J. 1993, 291, 139-144.

52. Schmidt, M.; Rutkat, K.; Rachel, R.; Pfeifer, G.; Jaenicke, R.; Viitanen, P.; Lorimer, G.; Buchner, J. Symmetric complexes of GroE chaperonins as part of the functional cycle. Science 1994, 265, 656-659. 
53. Corrales, F.J.; Fersht, A.R. Toward a mechanism for GroEL.GroES chaperone activity: an ATPase-gated and -pulsed folding and annealing cage. Proc. Natl. Acad. Sci. USA 1996, 93, 4509-4512.

54. Kawata, Y.; Nosaka, K.; Hongo, K.; Mizobata, T.; Nagai, J. Chaperonin GroE and ADP facilitate the folding of various proteins and protect against heat inactivation. FEBS Lett. 1994, 345, 229-232.

55. Sparrer, H.; Lilie, H.; Buchner, J. Dynamics of the GroEL-protein complex: effects of nucleotides and folding mutants. J. Mol. Biol. 1996, 258, 74-87.

56. Viitanen, P.V.; Donaldson, G.K.; Lorimer, G.H.; Lubben, T.H.; Gatenby, A.A. Complex interactions between the chaperonin 60 molecular chaperone and dihydrofolate reductase. Biochemistry 1991, 30, 9716-9723.

57. Hayer-Hartl, M.K.; Weber, F.; Hartl, F.U. Mechanism of chaperonin action: GroES binding and release can drive GroEL-mediated protein folding in the absence of ATP hydrolysis. EMBO J. 1996, 15, 6111-6121.

58. Staniforth, R.A.; Burston, S.G.; Atkinson, T.; Clarke, A.R. Affinity of chaperonin-60 for a protein substrate and its modulation by nucleotides and chaperonin-10. Biochem. J. 1994, 300, 651-658.

59. Kuwajima, K. The molten globule state of alpha-lactalbumin. FASEB J. 1996, 10, 102-109.

60. Martin, J.; Langer, T.; Boteva, R.; Schramel, A.; Horwich, A.L.; Hartl, F.U. Chaperoninmediated protein folding at the surface of groEL through a 'molten globule'-like intermediate. Nature 1991, 352, 36-42.

61. Semisotnov, G.V.; Rodionova, N.A.; Razgulyaev, O.I.; Uversky, V.N.; Gripas', A.F.; Gilmanshin, R. I. Study of the "molten globule" intermediate state in protein folding by a hydrophobic fluorescent probe. Biopolymers 1991, 31, 119-128.

62. Katsumata, K.; Okazaki, A.; Kuwajima, K. Effect of GroEL on the re-folding kinetics of alphalactalbumin. J. Mol. Biol. 1996, 258, 827-838.

63. Marchenkov, V.V.; Sokolovskii, I.V.; Kotova, N.V.; Galzitskaya, O.V.; Bochkareva, E.S.; Girshovich, A.S.; Semisotnov, G.V. The interaction of the GroEL chaperone with early kinetic intermediates of renaturing proteins inhibits the formation of their native structure. Biofizika 2004, 49, 987-994.

64. Perrett, S.; Zahn, R.; Stenberg, G.; Fersht, A.R. Importance of electrostatic interactions in the rapid binding of polypeptides to GroEL. J. Mol. Biol. 1997, 269, 892-901.

65. Hayer-Hartl, M.K.; Ewbank, J.J.; Creighton, T.E.; Hartl, F.U. Conformational specificity of the chaperonin GroEL for the compact folding intermediates of alpha-lactalbumin. EMBO J. 1994, 13, 3192-3202.

66. Katsumata, K.; Okazaki, A.; Tsurupa, G.P.; Kuwajima, K. Dominant forces in the recognition of a transient folding intermediate of alpha-lactalbumin by GroEL. J. Mol. Biol. 1996, 264, 643-649.

67. Marchenko, N.Y.; Marchenkov, V.V.; Kotova, N.V.; Kaliman, P.A.; Semisotnov, G.V. Complex of GroEL chaperone with fluorescein-labeled denatured pepsin: stoichiometry and role of the ligands. J. V.N. Karazin's Kharkov National Univ. 2005, 1, 53-56. 
68. Marchenko, N.I.; Marchenkov, V.V.; Kaisheva, A.L.; Kashparov, I.A.; Kotova, N.V.; Kaliman, P.A.; Semisotnov, G.V. Affinity chromatography of GroEL chaperonin based on denatured proteins: role of electrostatic interactions in regulation of GroEL affinity for protein substrates. Biochemistry (Moscow) 2006, 71, 1357-1364.

69. Viitanen, P.V.; Lubben, T.H.; Reed, J.; Goloubinoff, P.; O'Keefe, D.P.; Lorimer, G.H. Chaperonin-facilitated refolding of ribulosebisphosphate carboxylase and ATP hydrolysis by chaperonin 60 (groEL) are $\mathrm{K}^{+}$dependent. Biochemistry 1990, 29, 5665-5671.

70. Clark, A.C.; Frieden, C. GroEL-mediated folding of structurally homologous dihydrofolate reductases. J. Mol. Biol. 1997, 268, 512-525.

71. Ben-Zvi, A.P.; Chatellier, J.; Fersht, A.R.; Goloubinoff, P. Minimal and optimal mechanisms for GroE-mediated protein folding. Proc. Natl. Acad. Sci. USA 1998, 95, 15275-15280.

72. Weissman, J.S.; Hohl, C.M.; Kovalenko, O.; Kashi, Y.; Chen, S.; Braig, K.; Saibil, H.R.; Fenton, W.A.; Horwich, A.L. Mechanism of GroEL action: productive release of polypeptide from a sequestered position under GroES. Cell 1995, 83, 577-587.

73. Ying, B.W.; Taguchi, H.; Ueda, T. Co-translational binding of GroEL to nascent polypeptides is followed by post-translational encapsulation by GroES to mediate protein folding. J. Biol. Chem. 2006, 281, 21813-21819.

74. Zahn, R.; Buckle, A.M.; Perrett, S.; Johnson, C.M.; Corrales, F.J.; Golbik, R.; Fersht, A.R. Chaperone activity and structure of monomeric polypeptide binding domains of GroEL. Proc. Natl. Acad. Sci. USA 1996, 93, 15024-15029.

75. Picketts, D.J.; Mayanil, C.S.; Gupta, R.S. Molecular cloning of a Chinese hamster mitochondrial protein related to the "chaperonin" family of bacterial and plant proteins. J. Biol. Chem. 1989, 264, 12001-12008.

76. Nielsen, K.L.; Cowan, N.J. A single ring is sufficient for productive chaperonin-mediated folding In Vivo. Mol. Cell 1998, 2, 93-99.

77. Viitanen, P.V.; Lorimer, G.H.; Seetharam, R.; Gupta, R.S.; Oppenheim, J.; Thomas, J.O.; Cowan, N.J. Mammalian mitochondrial chaperonin 60 functions as a single toroidal ring. J. Biol. Chem. 1992, 267, 695-698.

78. Chatellier, J.; Hill, F.; Foster, N.W.; Goloubinoff, P.; Fersht, A.R. From minichaperone to GroEL 3: properties of an active single-ring mutant of GroEL. J. Mol. Biol. 2000, 304, 897-910.

79. Makino, Y.; Taguchi, H.; Yoshida, M. Truncated GroEL monomer has the ability to promote folding of rhodanese without GroES and ATP. FEBS Lett. 1993, 336, 363-367.

80. Taguchi, H.; Makino, Y.; Yoshida, M. Monomeric chaperonin-60 and its 50-kDa fragment possess the ability to interact with non-native proteins, to suppress aggregation; to promote protein folding. J. Biol. Chem. 1994, 269, 8529-8534.

81. Fenton, W.A.; Horwich, A.L. GroEL-mediated protein folding. Protein Sci. 1997, 6, 743-760.

82. Hartl, F.U.; Martin, J.; Neupert, W. Protein folding in the cell: the role of molecular chaperones Hsp70 and Hsp60. Annu. Rev. Biophys. Biomol. Struct. 1992, 21, 293-322.

83. Tang, Y.C.; Chang, H.C.; Roeben, A.; Wischnewski, D.; Wischnewski, N.; Kerner, M.J.; Hartl, F.U.; Hayer-Hartl, M. Structural features of the GroEL-GroES nano-cage required for rapid folding of encapsulated protein. Cell 2006, 125, 903-914. 
84. Tang, Y.C.; Chang, H.C.; Chakraborty, K.; Hartl, F.U.; Hayer-Hartl, M. Essential role of the chaperonin folding compartment in vivo. EMBO J. 2008, 27, 1458-1468.

85. Farr, G.W.; Fenton, W.A.; Horwich, A.L. Perturbed ATPase activity and not "close confinement" of substrate in the cis cavity affects rates of folding by tail-multiplied GroEL. Proc. Natl. Acad. Sci. USA 2007, 104, 5342-5347.

86. Kanno, R.; Koike-Takeshita, A.; Yokoyama, K.; Taguchi, H.; Mitsuoka, K. Cryo-EM structure of the native GroEL-GroES complex from Thermus thermophilus encapsulating substrate inside the cavity. Structure 2009, 17, 287-293.

87. Todd, M.J.; Lorimer, G.H.; Thirumalai, D. Chaperonin-facilitated protein folding: optimization of rate and yield by an iterative annealing mechanism. Proc. Natl. Acad. Sci. USA 1996, 93, 4030-4035.

88. Aoki, K.; Taguchi, H.; Shindo, Y.; Yoshida, M.; Ogasahara, K.; Yutani, K.; Tanaka, N. Calorimetric observation of a GroEL-protein binding reaction with little contribution of hydrophobic interaction. J. Biol. Chem. 1997, 272, 32158-32162.

89. Lin, Z.; Schwartz, F.P.; Eisenstein, E. The hydrophobic nature of GroEL-substrate binding. $J$. Biol. Chem. 1995, 270, 1011-1014.

90. Tsurupa, G.P.; Ikura, T.; Makio, T.; Kuwajima, K. Refolding kinetics of staphylococcal nuclease and its mutants in the presence of the chaperonin GroEL. J. Mol. Biol. 1998, 277, 733-745.

91. Corrales, F.J.; Fersht, A.R. The folding of GroEL-bound barnase as a model for chaperoninmediated protein folding. Proc. Natl. Acad. Sci. USA 1995, 92, 5326-5330.

92. Clark, A.C.; Hugo, E.; Frieden, C. Determination of regions in the dihydrofolate reductase structure that interact with the molecular chaperonin GroEL. Biochemistry 1996, 35, 5893-5901.

93. Deaton, J.; Sun, J.; Holzenburg, A.; Struck, D.K.; Berry, J.; Young, R. Functional bacteriorhodopsin is efficiently solubilized and delivered to membranes by the chaperonin GroEL. Proc. Natl. Acad. Sci. USA 2004, 101, 2281-2286.

94. Deaton, J.; Savva, C.G.; Sun, J.; Holzenburg, A.; Berry, J.; Young, R. Solubilization and delivery by GroEL of megadalton complexes of the lambda holin. Protein Sci. 2004, 13, 1778-1786.

95. Clark, A.C.; Ramanathan, R.; Frieden, C. Purification of GroEL with low fluorescence background. Methods Enzymol. 1998, 290, 100-118.

96. Voziyan, P.A.; Fisher, M.T. Chaperonin-assisted folding of glutamine synthetase under nonpermissive conditions: off-pathway aggregation propensity does not determine the cochaperonin requirement. Protein Sci. 2000, 9, 2405-2412.

97. Azem, A.; Kessel, M.; Goloubinoff, P. Characterization of a functional $\mathrm{GroEL}_{14}\left(\mathrm{GroES}_{7}\right)_{2}$ chaperonin hetero-oligomer. Science 1994, 265, 653-656.

98. Engel, A.; Hayer-Hartl, M.K.; Goldie, K.N.; Pfeifer, G.; Hegerl, R.; Muller, S.; da Silva, A.C.; Baumeister, W.; Hartl, F.U. Functional significance of symmetrical versus asymmetrical GroELGroES chaperonin complexes. Science 1995, 269, 832-836.

99. Llorca, O.; Carrascosa, J.L.; Valpuesta, J.M. Biochemical characterization of symmetric GroELGroES complexes. Evidence for a role in protein folding. J. Biol. Chem. 1996, 271, 68-76.

100. Sparrer, H.; Rutkat, K.; Buchner, J. Catalysis of protein folding by symmetric chaperone complexes. Proc. Natl. Acad. Sci. USA 1997, 94, 1096-1100. 
101. Todd, M.J.; Viitanen, P.V.; Lorimer, G.H. Dynamics of the chaperonin ATPase cycle: implications for facilitated protein folding. Science 1994, 265, 659-666.

102. Torok, Z.; Vigh, L.; Goloubinoff, P. Fluorescence detection of symmetric GroEL ${ }_{14}\left(\mathrm{GroES}_{7}\right)_{2}$ heterooligomers involved in protein release during the chaperonin cycle. J. Biol. Chem. 1996, 271, 16180-16186.

103. Marchenko, N.I.; Marchenkov, V.V.; Kotova, N.V.; Semisotnov, G.V.; Bulankina, N.I.; Kaliman, P.A. Effect of ADP and GroES on interaction of molecular chaperonin GroEL with non-native lysozyme. Ukr. Biokhim. Zh. 2003, 75, 88-94.

104. Plotnikov, A.N.; Vasilenko, K.S.; Kirkitadze, M.D.; Kotova, N.V.; Motuz, L.P.; Korotkov, K.V.; Semisotnov, G.V.; Alakhov, Y.B. Biosynthesis and conformational state of 17-kDa and 27-kDa N-terminal fragments of elongation factor EF-2 in solution. Bioorg. Khim. 1996, 22, 489-502.

105. Rye, H.S.; Burston, S.G.; Fenton, W.A.; Beechem, J.M.; Xu, Z.; Sigler, P.B.; Horwich, A.L. Distinct actions of cis and trans ATP within the double ring of the chaperonin GroEL. Nature 1997, 388, 792-798.

106. Burston, S.G.; Ranson, N.A.; Clarke, A.R. The origins and consequences of asymmetry in the chaperonin reaction cycle. J. Mol. Biol. 1995, 249, 138-152.

107. Ranson, N.A.; Burston, S.G.; Clarke, A.R. Binding, encapsulation and ejection: substrate dynamics during a chaperonin-assisted folding reaction. J. Mol. Biol. 1997, 266, 656-664.

108. Marchenkov, V.V.; Marchenko, N.I.; Marchenkova, S.Y.; Semisotnov, G.V. Molecular chaperonins of prokaryotic and eukaryotic cells. Adv. Biol. Chem.(Moscow) 2006, 46, 279-302.

109. Guex, N.; Peitsch, M.C. SWISS-MODEL and the Swiss-PdbViewer: an environment for comparative protein modeling. Electrophoresis 1997, 18, 2714-2723.

110. Grantham, J.; Llorca, O.; Valpuesta, J.M.; Willison, K.R. Partial occlusion of both cavities of the eukaryotic chaperonin with antibody has no effect upon the rates of beta-actin or alpha-tubulin folding. J. Biol. Chem. 2000, 275, 4587-4591.

111. Ehrnsperger, M.; Gaestel, M.; Buchner, J. Analysis of chaperone properties of small Hsp's. Methods Mol. Biol. 2000, 99, 421-429.

112. Markossian, K.A.; Yudin, I.K.; Kurganov, B.I. Mechanism of Suppression of Protein Aggregation by $\alpha$-Crystallin. Int. J. Mol. Sci. 2009, 10, 1314-1345.

113. Palleros, D.R.; Reid, K.L.; Shi, L., Welch, W.J.; Fink, A.L. ATP-induced protein-Hsp70 complex dissociation requires $\mathrm{K}^{+}$but not ATP hydrolysis. Nature 1993, 365, 664-666.

114. Wendler, P.; Shorter, J.; Snead, D.; Plisson, C.; Clare, D.K.; Lindquist, S.; Saibil, H.R. Motor mechanism for protein threading through Hsp104. Mol. Cell 2009, 34, 81-92.

(C) 2009 by the authors; licensee Molecular Diversity Preservation International, Basel, Switzerland. This article is an open-access article distributed under the terms and conditions of the Creative Commons Attribution license (http://creativecommons.org/licenses/by/3.0/). 